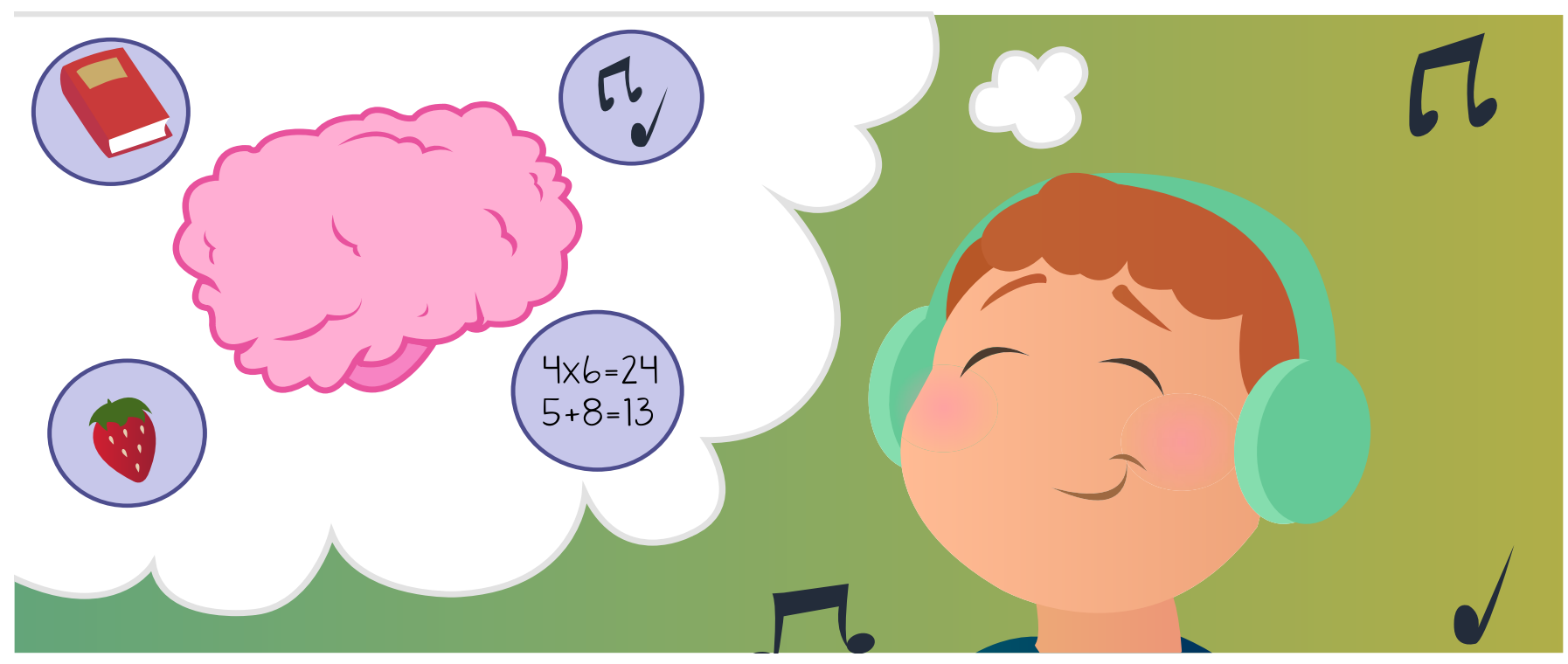

\title{
MUSIC: THE LAST THING WE FORGET
}

\section{Francine Foo ${ }^{1,2 *+}$ and Elizabeth L. Johnson ${ }^{1,3 *+}$}

${ }^{1}$ Helen Wills Neuroscience Institute, University of California, Berkeley, Berkeley, CA, USA, ${ }^{2}$ Biophysics Graduate Group, University of California, Berkeley, Berkeley, CA, USA, ${ }^{3}$ Department of Psychology, University of California, Berkeley, Berkeley, CA, USA

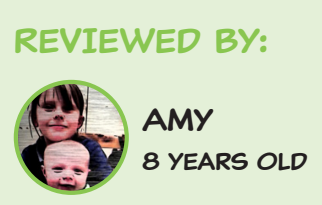

TTHESE AUTHORS HAVE CONTRIBUTED EQUALLY TO THIS WORK.
Have you ever wondered what happens in your brain when you think about your favorite songs? Recent research has revealed an area of the brain that is active when we listen to music that we know. This musical memory area is separate from the parts of your brain you use to remember things you have learned in school or details about events that happen in your life. In this article, we will show you where in the brain the musical memory area is, and why your memory for music is often resistant to brain diseases that cause memory loss.

\section{OUR MEMORY FOR MUSIC}

Try this simple exercise: go to your music library, pick a song, and play the first $3 \mathrm{~s}$ of it. Give yourself 1 point if you can manage to sing or hum at least the next $5 \mathrm{~s}$ of that song. Do this for 20 songs. How many points did you score? We would not be surprised if it is more than 15 . Now, think about what you just did. You effortlessly recalled the pitch, rhythm, and maybe even the lyrics of more than 15 songs in a short amount of time. That is a lot of data you managed to summon from your brain, just like that! 
Our brains possess a remarkable ability to make, store, and retrieve memories of music, even when we are not aware of doing so. For example, if you hear a catchy song, you will most likely be able to remember parts of it a few days later. After hearing it several times, you might know it by heart. Think about how much more effort it takes to learn information from a textbook, or to remember the details of day-to-day events in your life. Even more fascinating, musical memories seem to be very well preserved in people who suffer from amnesia-that is the clinical term for memory loss.

In his popular book Musicophilia, Dr. Oliver Sacks tells the story of musician and musicologist (i.e., an expert who studies music) Clive Wearing who, after suffering a devastating brain infection called herpes encephalitis, was unable to "retain an impression [memory] of anything for more than a blink" ([1], Chapter 15). Mr. Wearing was also unable to remember almost his entire past, but he could play pieces of music on the piano from memory and mouth the melodies while conducting a choir. The case of Mr. Wearing is not unique-non-musicians with severe amnesia can also show lasting memories of music ([1], Chapter 29). What, then, is so special about the ability of these people to remember music, even when they cannot remember just about anything else? In this article, we will show you where musical memories may be represented in the brain and how they can survive while other memories are lost.

\section{HOW DO MUSICAL MEMORIES DIFFER FROM OTHER LONG-TERM MEMORIES?}

In order to form and retrieve long-term memories (i.e., pieces of personal experiences and knowledge), multiple regions of the brain work together to form a coordinated network that transmits information from one brain region to another. For instance, when you remember what something you saw earlier that day looked like, you are using your occipital lobe, which is involved in vision. When you remember what you were thinking about earlier, or wonder how something that happened may have instead happened differently, you are using your frontal lobe (which is important for thinking). When you remember other moments in time, such as your own past (or when you think about your own future), you use multiple brain regions, including both the temporal and frontal lobes $[2,3]$. All of these different representations are put together in a specific region of the brain called the hippocampus, located within the temporal lobes, to form a memory.

Mr. Wearing's brain infection destroyed his hippocampus and other nearby brain regions, causing amnesia. The famous patient Henry Molaison, known by his initials as "H.M.," also suffered severe memory loss after doctors surgically removed his hippocampus and the tips of both temporal lobes (see Figure 1). By studying the cases of Mr. Wearing, H.M., and other 


\section{FIGURE 1}

Patient H.M.'s brain after surgery on the temporal lobes. The picture on the left shows a side view of the outside of the brain. The arrow is pointing at the tip of the temporal lobe. The picture on the right shows a crosssection of H.M.'s brain, from the viewpoint of someone looking up from his feet. The arrows are pointing to the bright portions of his temporal lobes, which are filled with fluid after removal of the brain tissue. Without these portions of the temporal lobes, H.M. could not form new memories or retrieve memories of events that happened in the years leading up to his surgery. Adapted from Ref. [2] with permission.
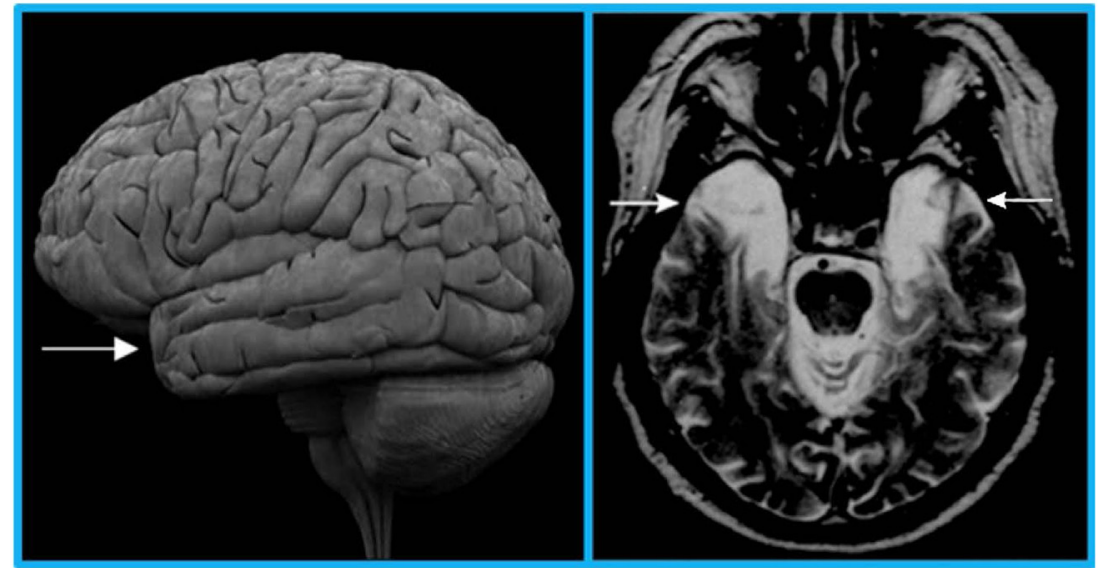

FIGURE 1

individuals with amnesia, we can conclude that the hippocampus and neighboring regions of the temporal lobe are crucial for creating and accessing long-term memories.

Another condition that causes damage to the hippocampus and nearby regions of the temporal lobe is dementia-a syndrome that affects a person's memory, thinking, and ability to interact with other people. In individuals with dementia, their cognitive abilities slowly start to decline. Beginning with mild amnesia, their condition gradually worsens over many years to the point where they can no longer care for themselves [3]. As the disease progresses, their brains show more and more damage throughout the network of temporal, frontal, and other regions involved in thinking about themselves [2-4].

Yet, despite profound memory loss and even a loss of knowledge about who they are, individuals with dementia often show a remarkable memory for music ([1], Chapter 29). Their musical memories somehow survive the widespread brain damage, even when other long-term memories do not. Might musical memories be processed somewhere else in the brain, in an area separate from the network of regions involved in long-term memory?

To answer this question, researchers recorded the brain responses of 32 healthy young adults as they listened to carefully pre-selected snippets of well-known, recently known, and completely unknown pieces of music [5]. The well-known pieces were selected from songs that were in the Top 10 between 1977 and 2007, nursery rhymes, and oldies, while the unknown songs were selected by looking at people's listening habits and choices on Amazon and Pandora. An hour before the participants' brains were scanned, they heard half of the songs in the unknown group twice, so that these songs would make up the "recently known" group. 


\section{FIGURE 2}

The "musical memory area" and its resistance to Alzheimer's disease (AD). This figure shows the brain as if it were cut down the middle, separating the left from the right half. Each row shows a picture of the left side of the brain from the inside (left) and outside (right) views. The top row displays the "musical memory area" (MMA) in red; it is also present in the same spot on the right side of the brain, which is not shown in this figure. In the second, third, and fourth rows, the MMA is shown with a white or black border (you will have to take a closer look to see it). The second, third, and fourth rows show the amount of three different kinds of damage in the brains of $A D$ patients: brain shrinkage, decreased glucose (sugar) uptake, and sticky buildup. You can see the amount of damage in the colored scale on the right side, with blue marking the least amount of damage, green marking some damage, and orange marking the most damage. Compared to other brain regions, the MMA shows the least damage from shrinkage and lack of glucose (marked in blue). Adapted from Ref. [5] with permission.
The researchers observed that two specific brain regions, called the ventral pre-supplementary motor area and the caudal anterior cingulate gyrus, were significantly more active when participants heard well-known songs compared to recently known or unknown songs. These brain regions are shown in red in the top row of Figure 2. Additionally, a computer could accurately predict whether a particular song was well-known, recently known, or unknown just by analyzing the patterns of brain activity in these regions. Taken together, the results of this study reveal a "musical memory area" (MMA) that enables us to remember our favorite songs. Importantly, this MMA is separate from the hippocampus and the temporal lobe that we know are necessary for long-term memory function.

\section{WHY MIGHT MUSICAL MEMORY BE PRESERVED IN PATIENTS WITH ALZHEIMER'S DISEASE (AD)?}

Now that we have identified the MMA, let us take a closer look at what happens in the brains of people who have $\mathrm{AD}$. $\mathrm{AD}$ is the most common

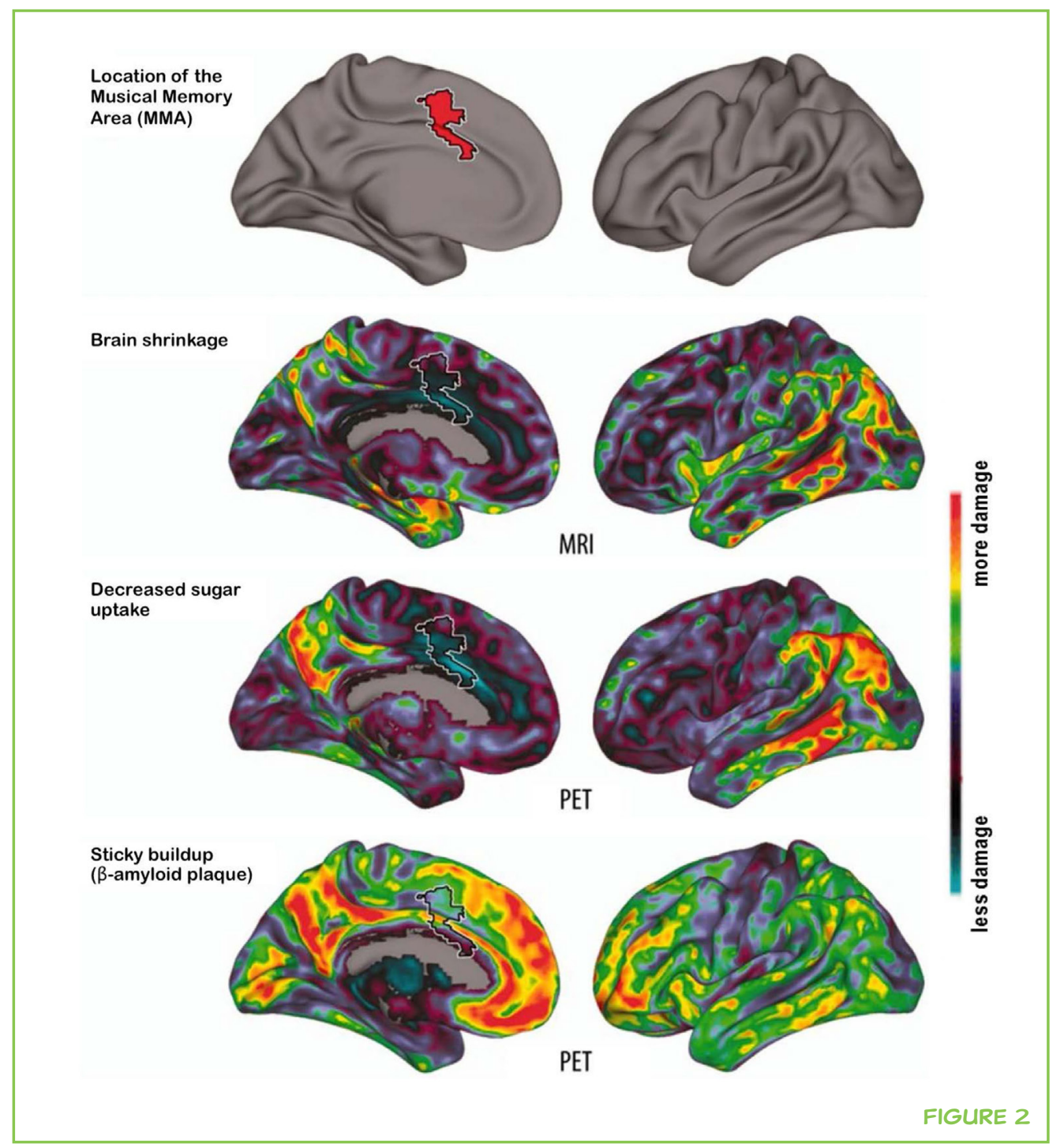


form of dementia, and it most frequently afflicts people close to your grandparents' age. People who suffer from AD start to lose their memories of who they are, where they have been, and what they have done. In later stages of the disease, they begin to lose their ability to speak, do simple everyday tasks, plan, solve problems, and interact well with other people. In other words, $\mathrm{AD}$ progresses beyond amnesia, impacting their feelings and knowledge about who they are and, eventually, every aspect of their lives $[2,3]$.

In order to diagnose $\mathrm{AD}$, doctors look for multiple symptoms in the brain. These include [4]:

(I) Shrinkage of the brain because brain cells are dying;

(II) An increase in a sticky buildup (of $\beta$-amyloid plaques) that causes brain cells to die; and

(III) A reduced amount of glucose (i.e., sugar) uptake, meaning that the brain is not consuming enough sugar to function properly.

To look for brain regions that have shrunk, doctors use a technique called magnetic resonance imaging (MRI) to scan the brain. To see the parts of the brain that have sticky buildup and/or are not getting enough sugar, doctors use a different technique called positron emission tomography (PET) to scan the brain. If you would like to learn more about $\mathrm{AD}$ and its diagnosis, check out Ref. [3].

This brings us to the second, third, and fourth rows of Figure 2. After identifying the MMA in healthy young adults (top row), the researchers took MRI and PET scans of 20 individuals with $A D$ who did not have musical training [5]. They found that the MMA showed some of the lowest amounts of shrinkage and it was still getting enough sugar to function properly (second and third rows, Figure 2). Unfortunately, the temporal lobes and the network of brain regions involved in long-term memory and self-knowledge are not so lucky. These regions are unable to consume enough sugar and suffer from shrinkage. If $\mathrm{AD}$ damages those regions and spares the MMA, it makes sense that musical memories survive while other long-term memories, as well as your feelings and knowledge about who you are, are lost.

Interestingly, the amount of sticky buildup in the MMA was not significantly lower than in other parts of the brain (fourth row, Figure 2) [5]. AD progression typically goes from sticky buildup $\rightarrow$ decreased sugar uptake $\rightarrow$ shrinkage throughout the brain, with the sticky buildup sometimes appearing even before noticeable memory loss [4]. Therefore, the researchers reasoned that the MMA is actually not degenerating as quickly as the temporal lobes and other regions involved in long-term memory. In fact, the MMA is well preserved over the course of $\mathrm{AD}$ and is among the last brain regions to degenerate [4], even when $\mathrm{AD}$ has destroyed most other parts of the brain. 


\section{CONCLUSION}

The identification of the MMA is an important piece of scientific evidence that explains why musical memories can survive even when amnesia and dementia have set in. As described by Dr. Sacks [1], individuals who have lost their long-term memories may appear to be stuck in the present, having lost access to knowledge about themselves, but they can amaze us with their memories for music. If musical memories can outlast damage to the hippocampus and the network of temporal, frontal, and other brain regions, these memories must be different than other long-term memories. This explains why Dr. Sacks was able to witness "mute, isolated, confused individuals warm to music, recognize it as familiar, and start to sing and bond" ([1], Chapter 29) and supports the strange yet magical power of music in our lives.

\section{ACKNOWLEDGMENTS}

We extend huge thanks to Dr. Robert Knight. We would also like to thank Keith Johnson, Rafael Nadal, and the BNP Paribas Open 2016 for inspiring the conceptualization of this article.

\section{REFERENCES}

1. Sacks, O. 2008. Musicophilia: Tales of Music and the Brain. New York: Vintage Books.

2. Dickerson, B. C., and Eichenbaum, H. 2010. The episodic memory system: neurocircuitry and disorders. Neuropsychopharmacology 35(1):86-104. doi:10.1038/npp.2009.126

3. Jagust, W. 2014. Dad can do time travel ... but grandpa can't! Front. Young Minds 2:18. doi:10.3389/frym.2014.00018

4. Benzinger, T. L. S., Blazey, T., Jack, C. R., Koeppe, R. A., Su, Y., Xiong, C., et al. 2013. Regional variability of imaging biomarkers in autosomal dominant Alzheimer's disease. Proc. Natl. Acad. Sci. U.S.A. 110:E4502-9. doi:10.1073/ pnas. 1317918110

5. Jacobsen, J. H., Stelzer, J., Fritz, T. H., Chételat, G., La Joie, R., and Turner, R. 2015. Why musical memory can be preserved in advanced Alzheimer's disease. Brain 138:2438-50. doi:10.1093/brain/awv135

SUBMITTED: 04 May 2016; ACCEPTED: 20 February 2017; PUBLISHED ONLINE: 13 March 2017.

EDITED BY: Robert T. Knight, University of California Berkeley, USA

CITATION: Foo F and Johnson EL (2017) Music: The Last Thing We Forget. Front. Young Minds 5:5. doi:10.3389/frym.2017.00005 
CONFLICT OF INTEREST STATEMENT: The authors declare that the research was conducted in the absence of any commercial or financial relationships that could be construed as a potential conflict of interest.

COPYRIGHT () 2017 Foo and Johnson. This is an open-access article distributed under the terms of the Creative Commons Attribution License (CC BY). The use, distribution and reproduction in other forums is permitted, provided the original author(s) or licensor are credited and that the original publication in this journal is cited, in accordance with accepted academic practice. No use, distribution or reproduction is permitted which does not comply with these terms.

\section{REVIEWED BY}

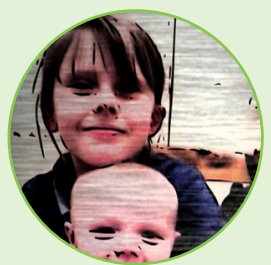

\section{AMY, 8 YEARS OLD}

I love gymnastics and playing on my tablet. My mummy is a psychologist and my daddy works for a company that makes machines for brain surgeons. I have a friend who is a brain surgeon too. I have a little brother who is four and a half months old. I love my whole family and I like to read.

\section{AUTHORS}

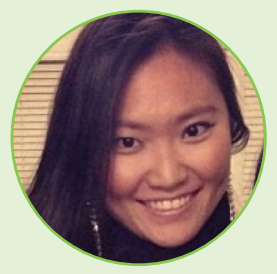

\section{FRANCINE FOO}

While I sometimes wonder what my life would have been like if I had pursued a career in music, I do not regret devoting the past 6 years to studying it. I seek to understand how the millions of cells in our brain interact as we perceive and enjoy the art form that is able to make us feel good, and at the same time compel us to get up on our feet and dance. When I am not hard at work, I secretly aspire to be a professional Latin Ballroom dancer.

*francinefoo@berkeley.edu

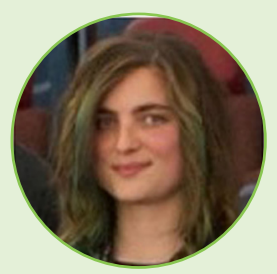

\section{ELIZABETH L. JOHNSON}

I find it incredible that this organ in our heads can make us so smart and so human. I want to understand memory - which allows us to think about so much information and put it all together, and craft identities based on personal experience. When I am not thinking about thinking, I like to explore weird and beautiful places and share experiences with weird and beautiful people. *eljohnson@berkeley.edu 